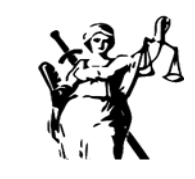

JUSTICIA

ISSN impreso 0124-7441
ISSN digital 2590-4566

\title{
Estrategias de ciudadanía juvenil aplicadas en la formación de agentes de Paz y Derechos Humanos
}

\section{Youth citizenship strategies applied in the training of Peace and Human Rights agents}

\author{
(iD) \\ Jorge Luis Restrepo Pimienta \\ Universidad del Atlántico, Colombia \\ jorgerestrepo@mail.uniatlantico.edu.co
}

\author{
Yamid Enrique Cotrina Gulfo \\ Universidad del Atlántico, Colombia \\ ycotrina@mail.uniatlantico.edu.co
}

\author{
(iD) \\ Alberto José Daza Sagbini \\ Universidad del Norte, Colombia \\ jaldaza@hotmail.com
}

Recibido: 24 de Agosto de 2020 / Aceptado: 30 Agosto de 2020 https://doi.org/10.17081/just.25.38.4455

\begin{abstract}
Resumen
Objetivo: Ejecutar estrategias de ciudadanía juvenil como herramienta de formación de agentes de paz en las Instituciones Educativas Distritales de la localidad suroriente de Barranquilla. Metodología: Se exponen los resultados de la ejecución del presente proyecto en la IED San José de Barranquilla, consistentes en el desarrollo de proyectos de servicio social obligatorio por parte de estudiantes de educación media de 10ㅡ, articulando iniciativas desde la Universidad y el Estado. Resultados y Conclusiones: Se llevaron a cabo talleres que en su conjunto corresponden a la formación en educación continuada en la modalidad de acción de formación a la población seleccionada, con la finalidad de fortalecer la Institución Educativa como espacio protector-promotor de paz y la reconciliación.
\end{abstract}

Palabras clave: agentes de paz, ciudadanía juvenil, consultorios jurídicos, derecho social comunitario, instituciones educativas.

\begin{abstract}
Objective: To execute youth citizenship strategies as a training tool for peace agents in the District Educational Institutions of the southeastern city of Barranquilla. Methodology: The results of the execution of this project in the IED San José de Barranquilla are presented, consisting of the development of mandatory social service projects by 10th grade students, articulating initiatives from the University and the State. Results and Conclusions: Workshops were carried out that together correspond to the training in continuing education in the Diploma modality to the selected population, with the purpose of strengthening the Educational Institution as a protective-promoter space of peace and reconciliation.
\end{abstract}

KEYWORDS: agents of peace, community social law, educational institutions, legal offices, youth citizenship.

\section{Como Citar:}

Restrepo Pimienta, J. L., Cotrina Gulfo, Y. E., \& Daza Sagbini , A. J. (2020). Estrategias de ciudadanía juvenil aplicadas en la formación de agentes de Paz y Derechos Humanos. Justicia, 25(38), 1-12. https://doi.org/10.17081/just.25.38.4455

* Artículo de investigación científica presentado como resultado final del proyecto de investigación e innovación "Impulso a Semilleros de Investigación del Programa de Derecho por áreas disciplinares, articulados a las líneas de investigación y a los Grupos de Investigación. 


\section{Introducción}

El goce efectivo de los derechos consagrados en el ordenamiento jurídico colombiano para los jóvenes, constituye a ser la problemática principal en la que se centran las actuales desigualdades en este sector poblacional. Mediante la elaboración de acuerdos sociales incluyentes, la gestión pacifica de los conflictos y la relación con el Estado e inclusión en Políticas Públicas con incidencia directa en el mejoramiento de sus condiciones de vida. Se propone diseñar estrategias que sirvan como espacios de empoderamiento a través del ejercicio de la ciudadanía juvenil como estrategia para la construcción de paz.

La formulación de proyectos e iniciativas por parte de los jóvenes, propicia la gobernanza y la conformación de una visión de desarrollo tomando como referente su progreso deseado en asuntos como participación ciudadana, salud, educación, seguridad personal y protección contra la violencia y discriminación, desde una perspectiva interseccional (mediante enfoques de género, de vulnerabilidad, étnico y los demás que haya lugar).

El enfoque regional en la ejecución del presente proyecto se evidencia en la incorporación del diagnóstico contenido en la Política Pública Departamental de Juventudes del Atlántico, escenario de planeación local y participativa y vehículo articulador para la consolidación del Estado Social de Derecho. Todo esto, con el fin de profundizar la relación con el Estado e inclusión de la juventud en la formulación de políticas públicas.

Lo anterior se ejecutará con fundamento en los siguientes Objetivos de Desarrollo Sostenible, seleccionados de manera prioritaria:

» ODS 5 Lograr la igualdad entre los géneros y empoderar a todas las mujeres y niñas: Se prevé como estrategia, la conformación de comités de apoyo e impulso en el proyecto la selección de no menos del $50 \%$ de la participación de las jóvenes, garantizando que ellas asuman posiciones de liderazgo y decisión.

\# ODS 10 Reducir la desigualdad en y entre los países: Aplicado este principio de manera específica a los grupos poblacionales, los jóvenes son entendidos como sujetos de vulnerabilidad social, por las condiciones de acceso a programas sociales en aras de democratizar sus derechos reconocidos.

$\gg$ ODS 16 Promover sociedades justas, pacificas e inclusivas: En la formulación de Políticas Públicas y el seguimiento de las ya vigentes se efectuará control social a las entidades responsables de su ejecución mediante acciones de la sociedad civil, producto de la formación jurídica para la exigibilidad de derechos.

$\gg$ ODS 17 Revitalizar la alianza mundial para el Desarrollo Sostenible: La alianza multisectorial es vista en este orden desde la Universidad del Atlántico como agente difusor del conocimiento, empleando sus procesos de extensión como el Consultorio Jurídico de la Facultad de Ciencias Jurídicas, en el cual estudiantes de últimos semestres de formación apoyarán el proceso de apropiación social del conocimiento, en la capacitación y entrenamiento a los estudiantes de Instituciones Educativas de la localidad suroriente de Barranquilla en ciudadanía juvenil, aplicando el derecho desde una perspectiva social y comunitaria.

Las Instituciones Educativas Distritales de la localidad suroriente de Barranquilla como entidades en la que estudiantes de educación media de 10으 desarrollan el servicio social obligatorio mediante proyectos de competencias ciudadanas como requisito de grado. Es contemplado como el escenario propicio para la articulación iniciativas por parte de los estudiantes de Semilleros de Investigación vinculados al Consultorio Jurídico de la Universidad del Atlántico para que no solo transmitan conocimiento con el fin de empoderar 
a los jóvenes en la exigibilidad de sus derechos, también la experiencia de alianza Universidad-Institución Educativa-Estado, puede traer consigo sinergias significativas que redunden en la generación de productos de investigación como ponencias a presentar en eventos de difusión del conocimiento. Se seleccionará exclusivamente al grado 10 para garantizar la ejecución del proyecto en el grado 11o en términos de medición de impacto del proyecto. Lo anterior se ejecutará con el propósito de fortalecer la institución educativa como espacio protector-promotor de paz y la reconciliación.

En primer orden, se relacionarán las estrategias de ciudadanía juvenil formuladas como resultado de la articulación de actividades de formación en catedra de paz entre Universidad, Institución Educativa y Estado como actores intervinientes del proyecto. En el siguiente segmento, se sistematizan las actividades de formación jurídica de las que fueron objeto los estudiantes de la Institución Educativa Distrital San José ubicada en la localidad suroriente de Barranquilla en mecanismos de protección y aplicación de derechos.

Finalmente, se describe el proceso de consolidación de liderazgos juveniles mediante acciones jurídico-pedagógicas tendientes a proyectar la participación de los estudiantes de educación media en escenarios de decisión, como es el caso de los órganos colegiados de la Institución Educativa, Organizaciones de la Sociedad Civil y Consejos Distritales y/o departamentales de juventudes.

\section{Metodología}

El proyecto se centra en el eje estratégico de promoción de la cultura de paz y reconciliación del CONPES 3850 de 2016 "Fondo Colombia en Paz", a través de la apropiación social del conocimiento como actividad principal de Ciencia Tecnología e Innovación dispuesta por COLCIENCIAS. Desde la ejecución de talleres, encuentros y diplomados como programas de formación siguiendo los ejes temáticos contenidos en las fases descritas a continuación:

TABLA 1.

RELACIÓN DE PRODUCTOS Y RESULTADOS DE INVESTIGACIÓN

\begin{tabular}{|c|c|c|c|}
\hline Fase & Producto & Resultado & Factor \\
\hline Preliminar & $\begin{array}{l}\text { Estrategias de ciudadanía } \\
\text { juvenil de formación en catedra } \\
\text { de paz }\end{array}$ & $\begin{array}{l}\text { Articulación entre Universidad } \\
\text { Institución Educativa Estado }\end{array}$ & Formación en la investigación \\
\hline Socialización & $\begin{array}{l}\text { Términos de ejecución del } \\
\text { proyecto }\end{array}$ & $\begin{array}{l}\text { Encuentro con docentes y } \\
\text { estudiantes }\end{array}$ & Formación de alto nivel \\
\hline Formación & $\begin{array}{l}\text { Diplomado en Ciudadanía } \\
\text { Juvenil como estrategia de } \\
\text { construcción de paz }\end{array}$ & $\begin{array}{l}\text { Formación en ciudadanía } \\
\text { juvenil }\end{array}$ & Formación continuada \\
\hline Proyección Social & Escuela de líderes & $\begin{array}{l}\text { Proyectar participación de } \\
\text { jóvenes a escenarios de partici- } \\
\text { pación ciudadana }\end{array}$ & Ciudadanía juvenil \\
\hline $\begin{array}{l}\text { Participación en eventos } \\
\text { científicos }\end{array}$ & $\begin{array}{l}\text { Experiencia: “Mi escuela territo- } \\
\text { rio de paz" }\end{array}$ & $\begin{array}{l}\text { Acciones de construcción de paz } \\
\text { en la IED San José }\end{array}$ & $\begin{array}{l}\text { Apropiación social del cono- } \\
\text { cimiento }\end{array}$ \\
\hline \multirow{2}{*}{$\begin{array}{l}\text { Producción académica } \\
\text { Proyección social }\end{array}$} & Artículo en revista indexada & Producción bibliográfica & \multirow[t]{2}{*}{ Difusión del conocimiento } \\
\hline & $\begin{array}{l}\text { Informe técnico de aplicación } \\
\text { para ONGs }\end{array}$ & $\begin{array}{l}\text { Perspectiva de efectividad en el } \\
\text { goce de derechos }\end{array}$ & \\
\hline
\end{tabular}

Fuente: Elaborada por los autores: 


\section{Estrategias de ciudadanía juvenil para la articulación de actividades de formación en Catedra de la Paz entre Universidad-Institución Educativa-Estado}

Consiste en un programa de extensión compuesto de la siguiente propuesta curricular que incluirá a fin de desarrollar competencias de Derecho Social Comunitario, mediante una acción de formación dirigida a estudiantes de 10 grado en educación media, que tendrá como espacio de implementación la institución educativa distrital San José de Barranquilla. Las actividades contenidas en el proyecto de apropiación social del conocimiento, cuyo contexto será el servicio social obligatorio dirigido a los estudiantes de educación media. El derecho social comunitario consiste en una aplicación epistémica de un campo de estudio propio de la psicología al derecho, enfatizando en la función social que tienen los juristas y la utilidad práctica que tienen sus conocimientos en la sociedad (Lorente Molina and Zambrano 2010). En la articulación con la formación de pregrado de estudiantes de derecho, se contempla como el primer nivel de práctica profesional en la modalidad de consultorio jurídico en escenarios como la apropiación social del conocimiento en comunidades priorizadas en la proyección social universitaria.

Para el caso concreto, los estudiantes de educación media, de decimo grado, fueron los seleccionados para garantizar así la puesta en marcha de los conocimientos adquiridos y el relevo generacional en los estudiantes de grados predecesores en nivel de formación. Con el fin de garantizar la correcta aplicación de los objetivos del proyecto, se contempla como primera la fase preparatoria, en la que se concertará con las autoridades de la Universidad del Atlántico e Institución Educativa en aras de completar la alianza Universidad-Institución Educativa y Estado, este último interviene en la aprobación, financiación y asistencia técnica del proyecto.

TABLA 2:

FASE DE FORMULACIÓN Y PREPARACIÓN DE ESTRATEGIAS

\begin{tabular}{|c|c|}
\hline Actividad & Contenido \\
\hline $\begin{array}{l}\text { Diseñar plan de capacitación jurídica desde el Consultorio } \\
\text { Jurídico hacia los estudiantes de educación media. }\end{array}$ & $\begin{array}{l}\text { Plan de capacitación jurídica en mecanismos de protección de derechos: } \\
\text { Derecho de Petición. } \\
\text { Acciones de Tutela. } \\
\text { Acciones de Cumplimiento. } \\
\text { Acciones de Grupo. } \\
\text { Acciones Populares. }\end{array}$ \\
\hline $\begin{array}{l}\text { Formular proyectos de Competencias Ciudadanas a eje- } \\
\text { cutar en Instituciones Educativas Distritales por parte de } \\
\text { docentes y estudiantes de educación media. }\end{array}$ & $\begin{array}{l}\text { Proyecto de competencias ciudadanas con énfasis en ciudadanía juvenil } \\
\text { en el que desarrollen los siguientes ámbitos: } \\
\text { Gestión pacífica de conflictos. } \\
\text { Formulación de proyectos. } \\
\text { Goce efectivo de derechos. } \\
\text { Interseccionalidad. }\end{array}$ \\
\hline $\begin{array}{l}\text { Elaborar un plan de articulación en política juvenil entre } \\
\text { la gobernación del Atlántico y la Universidad del Atlántico } \\
\text { aplicándose en Instituciones Educativas Distritales. }\end{array}$ & $\begin{array}{l}\text { Plan de articulación en política pública juvenil con los siguientes compo- } \\
\text { nentes: } \\
\text { Igualdad de género. } \\
\text { Enfoque de vulnerabilidad. } \\
\text { Ciudadanía juvenil. }\end{array}$ \\
\hline $\begin{array}{l}\text { Encuentro con docentes y directivos docentes del plantel } \\
\text { educativo. }\end{array}$ & $\begin{array}{l}\text { Acordar los términos de ejecución del proyecto, cronogramas y metod- } \\
\text { ologías. }\end{array}$ \\
\hline $\begin{array}{l}\text { Encuentro con estudiantes de } 10 \text { o grado de Instituciones } \\
\text { Educativas Distritales }\end{array}$ & $\begin{array}{l}\text { Seleccionar un grupo no mayor a treinta (30) estudiantes (de los } 135 \text { ma- } \\
\text { triculados), para iniciar el proceso de formación en ciudadanía juvenil. }\end{array}$ \\
\hline
\end{tabular}

Fuente: Elaborada por los autores. 
Los proyectos formativos deben responder a los retos actuales en materia de juventud y propender por acciones de inclusión social, a través de la gestión de conflictos, formulación de proyectos, goce efectivo de derechos y la Interseccionalidad. Estos objetivos se tienen en cuenta de manera sucesiva en el siguiente entendido:

$\gg$ Formular proyectos que contengan iniciativa de innovación social

» Formación jurídica para garantizar el acceso a la justicia y la exigibilidad de derechos

» Interseccionalidad para aplicar los componentes temáticos desde un enfoque poblacional, de vulnerabilidad y en derechos humanos teniendo en cuenta las particularidades del contexto a aplicarse.

Según Patero, la interseccionalidad "es un enfoque que subraya que el género, la etnia, la clase u orientación sexual, como otras categorías sociales, lejos de ser "naturales" o "biológicas", son construidas y están interrelacionadas (Patero Mendez 2014). Esto hace referencia a las anteriores particularidades mencionadas que pueden conformar la población, esto debe tenerse en cuenta para garantizar la pertinencia de la acción a implementar con el proyecto de apropiación social a formular. Para garantizar la exigibilidad de derechos, se debe tener en cuenta el recurso y la naturaleza del recurso a impetrar al igual que el bien jurídico vulnerado, el cual se pretender resarcir con la acción.

Resultados de la primera fase: Se acordó con la directiva de la institución la capacitación mediante acción de formación suministrada por la Universidad del Atlántico certificada a los estudiantes de $10^{\circ}$ (decimo grado) en ciudadanía juvenil como estrategia para la construcción de paz. El mecanismo de implementación será blended learning, o metodología mixta de base virtual, con actividades presenciales complementarias.

\section{Formación jurídica en mecanismos de protección y aplicación de derechos}

Se diseñó un plan de estudios y contenido programático de las sesiones y articulación con programas curriculares del aula. Entre el contenido curricular se destaca la importancia de la ética, democracia y la educación como elementos indispensables para el Estado de Derecho sustentan valores éticos para la educación y la ciudadanía como la dignidad humana, la justicia, la seguridad, la tolerancia, el pluralismo, la solidaridad y la correcta relación entre la libertad y la igualdad. La relación entre derechos y deberes suponen una relación entre el individuo y sus semejantes, lo cual supone un reto para la protección y garantía de los derechos fundamentales. La relación entre las personas y las instituciones como garantes de los derechos humanos suponen un ejercicio del derecho y del poder adecuadamente en una sociedad democrática (Peces Barba et al. 2007) (Peces Barba et al. 2007).

El proceso de selección de la plataforma virtual para cursos e-learning o b-learning es una de las tareas más importantes, en cuanto que nos delimitará y marcará las metodologías pedagógicas que se pueden desarrollar en función de las herramientas y servicios que ofrezcan. El ambiente de aprendizaje se crea sobre las plataformas, de modo que estas deben disponer de los elementos que consideremos necesarios para un aprendizaje de calidad, en el que los alumnos puedan construir sus conocimientos, comunicándose y colaborando con profesores y otros alumnos (Belloch 2012).

Se elabora material audiovisual para la elaboración del curso que, de acuerdo con la vicerrectoría de investigaciones en su dependencia de extensión y proyección social, recomiendan que esta actividad por ser propia de la proyección social se haga no como diplomado sino como acción de formación virtual, que se desarrollará a través de la plataforma virtual SICVI-567. Mientras se realizan las gestiones con vicerrectoría de docencia para habilitar el espacio en la plataforma se procede a grabar los vídeos para alojarlos en el sitio web que será de acceso para los estudiantes seleccionados con anterioridad. 
Se hace envío al docente del material que se va a subir a la plataforma: vídeos, talleres evaluativos, material de lectura correspondientes a las lecciones, quien da su aprobación al contenido programático al igual que a los contenidos del curso virtual.

La modalidad virtual de la acción de formación, con actividades presenciales a ejecutar de manera conjunta con los docentes de la institución educativa distrital san José. Hace que los talleres sean impartidos y las actividades presenciales como talleres y encuentros lúdicos, sean llevados a cabo de manera conjunta con la institución educativa. Se cumplen de igual manera con los protocolos de manejo y protección de datos y toma de muestras audiovisuales con menores de edad al igual que se fija la estructura de costos, aprobación y asistencia técnica con la vicerrectoría de investigaciones de la Universidad del Atlántico.

De esta manera, se articula el cumplimiento de los procesos entre Universidad y Sociedad (en este caso, institución educativa como destinataria del proyecto de apropiación social del conocimiento). El Estado, entra a la sinergia en el diseño de protocolos de acuerdo al CONPES 3850 de 2016 "Fondo Colombia en Paz".

Las ventajas de seleccionar la plataforma MOOC para el desarrollo del curso consiste en: Permitir el diseño de la actividad colaborativa; es decir, más que la implementación de un ambiente colaborativo específico, debe proveer las herramientas para el diseño y construcción de ambientes colaborativos flexibles que sean modificables y explorables por el grupo. En relación al diseño de la actividad debe involucrar al grupo de participantes y los motive a la exploración y participación activa. Los participantes entrarán a formar parte activa dentro de la actividad como protagonistas y actores (Mendoza and Galvis 1999).

Google es sinónimo de innovación y está expandiendo las posibilidades de acceso a la información sin precedentes en la historia de la humanidad. Con una presencia muy fuerte en la WWW y ahora brindando las mismas posibilidades desde la telefonía móvil, se considera un gran aliado para utilizarse con fines educativos (González Pérez 2011).

Las unidades temáticas incluidas en la acción de formación en ciudadanía juvenil como estrategia de construcción de paz dirigida a los estudiantes enunciados con anterioridad contienen los siguientes elementos.

ODS y juventud: Los objetivos de desarrollo sostenible son entendidos para el contexto del presente proyecto como la meta de todos los países que forman la ONU (Organización de las Naciones Unidas), para cumplir el Derecho al Desarrollo, este es entendido como la satisfacción de las necesidades básicas. Si se quiere decir de otra manera, el derecho al desarrollo puede entenderse realizado cuando todas las personas cuenten con un nivel de vida adecuado (Obando 2003). Esto último es objeto de múltiples debates, toda vez que su respuesta es tan variada por tantas personas la formulen (Escobar 2002). La cultura influye mucho en esta percepción y por lo tanto hay que tener en cuenta los valores y principios de cada una de las personas y de sus comunidades, porque la multiplicidad de formas de ver la vida es una fuente de riqueza social.

Mecanismos de protección y aplicación de derechos: Supone un especial reto la exigibilidad de derechos sociales, puesto que no representan derechos subjetivos sin sí (Spino Tapia 2017) (Gulfo and Yamid Enrique 2020). Estos mecanismos como el derecho de petición como medida inicial y la acción de tutela, desde un sentido individual en el reconocimiento de derechos fundamentales y de aquellos que la constitución reconoce como aquellos de inmediata aplicación en su artículo 85. Al igual que aquellas acciones colectivas que se interponen con una finalidad indemnizatoria (acción de grupo) o preventiva (acción popular). 


\section{TABLA 3:}

UNIDADES TEMÁTICAS DE LA ACCIÓN DE FORMACIÓN

\begin{tabular}{|c|c|}
\hline Actividad & Contenido \\
\hline $\begin{array}{l}\text { Primer ciclo de talleres. } \\
\text { Taller 1: Ciudadanía juvenil: Objetivos de } \\
\text { Desarrollo Sostenible y Juventud. }\end{array}$ & $\begin{array}{l}\text { Formación teórica en Objetivos de Desarrollo Sostenible aplicados a la juventud para } \\
\text { treinta (30) estudiantes. }\end{array}$ \\
\hline $\begin{array}{l}\text { Taller 2: Mecanismos de protección } \\
\text { y aplicación de derechos (Primera } \\
\text { sección). }\end{array}$ & $\begin{array}{l}\text { Formación desde el consultorio jurídico de la Universidad del Atlántico en mecanismos } \\
\text { de protección de derechos: } \\
\text { Derecho de Petición. } \\
\text { Acciones de tutela. } \\
\text { Acciones de cumplimiento. } \\
\text { Habeas Corpus }\end{array}$ \\
\hline $\begin{array}{l}\text { Taller 3: Mecanismos de protección } \\
\text { y aplicación de derechos (Segunda } \\
\text { sección). }\end{array}$ & $\begin{array}{l}\text { Formación jurídica en la formulación de mecanismos de protección y aplicación de } \\
\text { derechos, como: } \\
\text { Acciones de tutela. } \\
\text { Acciones populares. } \\
\text { Acciones de Grupo. }\end{array}$ \\
\hline $\begin{array}{l}\text { Taller 4: Normas jurídicas y políticas } \\
\text { públicas y juventud. }\end{array}$ & $\begin{array}{l}\text { Formación teórica en políticas públicas, formulación, ejecución y rendición de cuentas. } \\
\text { Constitución Política de Colombia } \\
\text { Tratados Internacionales } \\
\text { Código de Infancia y Adolescencia } \\
\text { Código de Policía }\end{array}$ \\
\hline $\begin{array}{l}\text { Taller 5: Mecanismos de asociatividad } \\
\text { juvenil. }\end{array}$ & $\begin{array}{l}\text { Formación jurídica en organizaciones de la sociedad civil sin ánimo de lucro: } \\
\text { Fundaciones. } \\
\text { Corporaciones. } \\
\text { Asociaciones. } \\
\text { Clubes deportivos. } \\
\text { Plataformas de juventudes. }\end{array}$ \\
\hline $\begin{array}{l}\text { Taller 6: Entidades públicas vinculadas a } \\
\text { las políticas de juventudes. }\end{array}$ & $\begin{array}{l}\text { Formación jurídica en entidades gubernamentales de juventudes: } \\
\text { Ministerios. } \\
\text { Departamentos Administrativos. } \\
\text { Entes territoriales. }\end{array}$ \\
\hline Taller 7: Interseccionalidad y juventud. & $\begin{array}{l}\text { Formación teórica en aplicación de enfoques en política pública de juventud: } \\
\text { Enfoque de género. } \\
\text { Perspectiva de vulnerabilidad. } \\
\text { Enfoque diferencial. } \\
\text { Enfoque étnico. } \\
\text { Enfoque de derechos humanos. } \\
\text { Enfoque de desarrollo humano. }\end{array}$ \\
\hline Taller 8: Mi escuela territorio de paz. & $\begin{array}{l}\text { Encuentro teórico-práctico en el que se fija el plan de acción de construcción de paz en } \\
\text { la Instituciones Educativas. Con las siguientes actividades: } \\
\text { Proyecto de vida } \\
\text { Declaración de ciudadanía juvenil. (Plan de acción en DD.HH.) } \\
\text { Partido de futbol por la paz (Herramientas de mediación) }\end{array}$ \\
\hline Certificación. & $\begin{array}{l}\text { Mediante la acción de formación en "Ciudadanía Juvenil como estrategia de construc- } \\
\text { ción de paz", la Universidad del Atlántico certificará a los estudiantes Instituciones Edu- } \\
\text { cativas Distritales de la localidad suroriente de Barranquilla. (Equivalentes a } 2 \text { créditos } \\
\text { académicos, } 96 \text { horas de formación) }\end{array}$ \\
\hline
\end{tabular}

Fuente: Elaborada por los autores. 
Todo esto se vincula necesariamente con la siguiente unidad denominada: Normas jurídicas y políticas públicas y juventud, en la que se fundamentan y/o desarrollan los derechos, garantías y libertades de los jóvenes en Colombia desde el plano legal, constitucional e internacional. En este mismo sentido se encuentran las Entidades públicas vinculadas a las políticas de juventudes. Desde los niveles municipal departamental, nacional e incluso internacional al igual que aquellas organizaciones de la sociedad civil referentes en materia de juventud que son garantes de los derechos de los jóvenes en cuanto a la formulación de planes, programas y proyectos de impacto poblacional, de igual manera se pretende fomentar un espacio de dialogo y concertación entre jóvenes y entidades responsables del diseño de política pública.

Mecanismos de asociatividad juvenil: Se reitera la importancia de la asociatividad entre los jóvenes mediante las redes y plataformas de juventudes como espacio de disertación y deliberación de problemáticas que afecten al grupo poblacional. De igual manera se enuncian las figuras jurídicas que contempla la legislación civil-comercial en Colombia para constituir Entidades Sin Ánimo de Lucro - ESAL, y sus elementos esenciales tal como se contempla en la normatividad vigente.

Interseccionalidad y juventud. La juventud como grupo social heterogéneo, se compone de múltiples poblaciones, por lo tanto, para garantizar la aplicación de la presente propuesta, se propone aplicar la igualdad entre hombres y mujeres en la acción de formación y que esta sea conducente para formar a la mujer como sujeto de cambio e incidencia social (enfoque de género), de igual manera se atenderá las particularidades de cada quien desde los criterios definidos en el artículo 13 de la constitución política incluyendo a todas las personas participantes y que sus modos de vida no sean incompatibles con esta iniciativa, sino que por el contrario, puedan ver en esta acción de formación un espacio para potencializar sus capacidades (perspectiva de vulnerabilidad y enfoque diferencial).

Se valorará la diversidad étnica y cultural como bien jurídicamente protegido en el ordenamiento jurídico y que las personas de grupos étnicos beneficiarios de enfoques diferenciales (afrodescendientes e indígenas en este caso), apliquen los conocimientos adquiridos en el beneficio de sus comunidades (enfoque étnico), al igual que favorezca la realización efectiva de los derechos de las personas y poblaciones (enfoque de derechos humanos). Con la garantía de derechos, se prevé que se genere inclusión en la formulación de política pública y así fomentar igualdad y llevar a la población a mejorar sus condiciones de vida al mayor nivel posible (enfoque de desarrollo humano).

Mi escuela territorio de paz: Actividad de socialización en la que mediante actividades artísticas y deportivas (para resaltar la importancia de los clubes deportivos como mecanismos de asociatividad juvenil). También se contempla como un espacio para el relevo generacional, en el que mediante tales jornadas se vinculen a los niños en grados iniciales de básica primaria y educación temprana para la formación en competencias ciudadanas. Insistiendo en la escuela como un espacio seguro, libre de violencia y toda forma de agresión. Se contó con el apoyo de docentes, estudiantes participantes del proyecto y personal de la Universidad del Atlántico.

\section{Consolidación de liderazgos juveniles en la participación de jóvenes en escenarios de decisión}

En este segundo segmento de la acción de formación, se hace énfasis en la formulación de proyectos como acción que direcciona las iniciativas tendientes a la formulación y desarrollo de política publica en materia de juventud. De igual manera, se hace un acercamiento al contexto a los jóvenes con organizaciones de la sociedad civil cuyas acciones sean tendientes a la mejora de las condiciones de vida y al fomento de la participación de los jóvenes en los espacios de decisión. 
TABLA 4:

RELACIÓN DE ACTIVIDADES PRÁCTICAS DE LA ACCIÓN DE FORMACIÓN

\begin{tabular}{|l|l|}
\hline \multicolumn{1}{|c|}{ Actividad } & \multicolumn{1}{c|}{ Contenido } \\
\hline $\begin{array}{l}\text { Segundo ciclo de talleres. } \\
\text { Taller 1: Formulación de proyectos. }\end{array}$ & $\begin{array}{l}\text { Taller teórico-práctico empleando la innovación social como perspectiva de for- } \\
\text { mación, en formulación de proyectos con énfasis en juventudes. }\end{array}$ \\
\hline $\begin{array}{l}\text { Salida de campo 1: Encuentro con la Plataforma } \\
\text { de juventudes. }\end{array}$ & Encuentro para socializar experiencias exitosas de empoderamiento juvenil. \\
\hline Taller 2: Formación en competencias políticas. & $\begin{array}{l}\text { Formación teórica en escenarios de participación ciudadana en asuntos de juventud, } \\
\text { componente práctico de expresión oral y manejo de público. }\end{array}$ \\
\hline $\begin{array}{l}\text { Salida de campo 2: Encuentro con Juntas de } \\
\text { Acción Comunal (JAC) }\end{array}$ & $\begin{array}{l}\text { Encuentro con miembros de la (JAC) para elaborar un diagnóstico de necesidades en } \\
\text { juventudes. }\end{array}$ \\
\hline Taller 3: Escuela de líderes. & $\begin{array}{l}\text { Encuentro con los jóvenes participantes en el proceso de formación para proyectar } \\
\text { su participación en escenarios de decisión juvenil en el siguiente orden: } \\
\text { Instituciones Educativas Distritales: Elección de Representante de los Estudiantes } \\
\text { ante el Consejo Directivo y de Personero. } \\
\text { Organizaciones de la Sociedad Civil: Procesos de liderazgos desde una perspectiva } \\
\text { poblacional. } \\
\text { Consejo Distrital y/o Departamental de Juventudes: Resultado a mediano plazo } \\
\text { del proceso de formación en ciudadanía juvenil en la participación política de los } \\
\text { jóvenes. }\end{array}$ \\
\hline
\end{tabular}

Fuente: Elaborada por los autores.

En esta parte del proceso de formación se hace énfasis en la formación en competencias políticas de los jóvenes para que estos accedan a las instancias de decisión y participación, en política juvenil, o cual se considera como el elemento esencial de la ciudadanía juvenil en el contexto del presente proyecto. Para ello se contemplan los siguientes ejes a fortalecer en la formación política de los jóvenes.

$\gg$ Ejes de direccionamiento político: Como motor de su carrera política: (denominarlo y construir un objetivo político) y líneas de acción programáticas. En lo que se considera como meta la política pública, que desarrolla el plan de desarrollo a nivel de ente territorial.

$\gg$ Eje social: Garantía de igualdad, en la formulación y ejecución política pública. Esto se contempla teniendo en cuenta los enfoques poblacionales a atender, puesto que la juventud es comprendida como un colectivo social heterogéneo. (ODS 10: Reducción de las desigualdades)

$\gg$ Eje económico: fortaleciendo las áreas de emprendimiento, trabajo decente, desarrollo económico juvenil, mayores políticas públicas sociales, potencializar la capacidad económica (ODS 8: Trabajo decente y crecimiento económico).

$\gg$ Eje institucional: Gerencia publica, buen gobierno, gobierno abierto, rendición de cuentas, acercar al gobierno, mayor control ciudadano, toma de decisiones. (ODS 16: Paz, justicia e instituciones sólidas).

$\gg$ Eje ambiental y territorial: conciencia del crecimiento atendiendo de manera paralela a la problemática que genera el cambio climático, erradicación del pensamiento de la desconexión entre el ambiente y político (ODS 11: Ciudades y comunidades sostenibles).

$\gg$ Focalización: En aras de entender las realidades y situaciones de un contexto en particular para definir las líneas de acción que tendrán impacto directo en su comunidad. Esto con el fin de generar en el estudiante la capacidad de construir una propuesta con pertinencia al contexto y esta pueda aplicarse de manera adecuada atendiendo a las particularidades del mismo. 
$\gg$ Capacidad diagnostica: Habilidad previa, decir que está pasando entender todos los contextos del problema. Diseñar los ejes del proyecto teniendo como base lo diseñado anteriormente, se formulan planes y se conectan con un presupuesto surgiendo la política pública. (documento o acción que desarrolla un eje o un parámetro de acción política que ha sido programada), con el fin de seguir con los procesos ya ejecutados.

$\gg$ Competencias comunicativas: Saber comunicar, construir equipos de trabajo, competencias escritas y en oratoria, diplomacia como competencia para saber negociar.

\section{Conclusiones}

La formación jurídica a estudiantes de niveles en educación media para fomentar el relevo generacional al interior de la institución y propiciar un ambiente escolar garante de los derechos humanos y educado en el respeto a la diferencia que conforma a todos los miembros de la Institución Educativa. Fue el objetivo que se logró llevar a cabo con las actividades de concertación, formación y aplicación del proyecto. Desde el acuerdo con los docentes y directivos docentes de la Institución Educativa, contando también con el acompañamiento y asistencia técnica de la Universidad y el Estado hicieron posible la realización de la experiencia formativa que tiene altas probabilidades de replicación.

Se dejó a disposición de la Institución Educativa el material de formación para que pueda ser empleado en las próximas generaciones y las actividades lúdicas para que los proyectos de servicio social de los estudiantes coadyuven a la formación de la escuela como un ambiente seguro para todos los estudiantes, libre de matoneo o bullying y en caso de llegar a presentarse, tomar los correctivos del caso.

El relevo generacional es fundamental, puesto que para el $10^{\circ}$ grado es la etapa de aprendizaje y en el $11^{\circ}$ grado, es la etapa de puesta en practica de lo aprendido en la acción de formación, lo cual garantiza una continuidad en los procesos de formación en competencias ciudadanas. Vincular el compromiso de los docentes de las áreas de Ciencias Sociales, fue fundamental puesto que la actividad extracurricular fue un indicador de evaluación a tener en cuenta de manera complementaria en la enseñanza.

La formación en ciudadanía juvenil, permite la generación de nuevos liderazgos, que fuera de la Institución Educativa, y en sus propios contextos puedan aplicar lo aprendido tanto desde lo local en las Juntas de Acción Comunal y dinamizar tal escenario de participación, como aquellos incidentes en la participación de los jóvenes como grupos poblacionales en las plataformas y los consejos de juventudes.

\section{Referencias}

Balaedini, Sergio. 2000. “De Los Jóvenes, La Juventud y Las Políticas de Juventud." Última Década 8(13):11-24. Belloch, Consuelo. 2012. Entornos Virtuales de Aprendizaje. Valencia.

Escobar, Arturo. 2002. “Globalización, Desarrollo y Modernidad. Planeación, Participación y Desarrollo." Corporación Región 9-32.

González Pérez, Esteban. 2011. "Recursos de Google Para El Desarrollo de Una Unidad Didáctica Con Estudiantes de Educación Superior.” Revista Electrónica» Actualidades Investigativas En Educación 11:1-15.

Gulfo, Cotrina and Yamid Enrique. 2020. "Necesidades Como Criterio Fundamentador de Los Derechos Humanos." UNIVERSITAS. Revista de Filosofía, Derecho y Política, Universidad Carlos III de Madrid (33):85-100. 
Lorente Molina, Belén and Carlos Zambrano. 2010. "Reflexividad, Trabajo Social Comunitario y Sensibilización En Derechos." Cuadernos de Trabajo Social (23):85-102.

Mendoza, Patricia and Álvaro Galvis. 1999. "Ambientes Virtuales de Aprendizaje: Una Metodología Para Su Creación." Revista Informática Educativa 12(2):295-317.

Obando, Álvaro. 2003. “Sentidos Sociales y Participativos de La Planeación Participativa." Pp. 11-51 in La planeación participativa, una apuesta de ciudad. Medellín: Corporación Región, CEDESIS, Convivamos, IPC, \& Fundación Social.

Patero Mendez, Raquel. 2014. “Metáforas y Articulaciones Para Una Pedagogía Crítica Sobre La Interseccionalidad." Quaderns de Psicología, Universidad Autónoma de Barcelona 16(1):55-72.

Peces Barba, Gregorio, Rafael De Asís, Eusebio Fernández, and Javier Francisco Ansuátegui. 2007. Educación Para La Ciudadanía y Derechos Humanos. Madrid: Ed. Espasa.

Spino Tapia, Diana Rocío. 2017. "'Derechos Sociales y Justiciabilidad En La Teoría Constitucional de Inicios Del Siglo XXI', Cuestiones Constitucionales." Revista Mexicana de Derecho Constitucional (36):83-104. 
